\section{ORGANIC SYMBOLS}

The idea to assign an individual symbol to each organic compound, of which over 110,000 are known, seems absurd, impractical and too complicated for a student to master. It would even put the Chinese characters in the background as far as the number of symbols is concerned. Nevertheless a system of organic symbols is not only possible and practical, but also simple and efficient and offers a timeand space-saving device for modern chemistry. In a recent paper ${ }^{1}$ such a system of symbols was outlined and some of its advantages have been pointed out. Each symbol represents the structure of the organic compound and indicates furthermore the optical activity, isomeric form and chemical type of a definite organic compound.

The system of symbols is based upon the four elements, hydrogen, oxygen, nitrogen and carbon, while all the other elements entering into a compound are represented by their ordinary chemical symbols. The atoms of these four elements are thought to be points in the symbols, these points to be determined by lines terminating $(\mathrm{H})$, meeting ( $\mathrm{O}$ and $\mathrm{N}$ ) or crossing (C). Accordingly hydrogen is a point from which one line radiates, oxygen a point from which two lines radiate, nitrogen three and carbon four lines, radiating respectively. The lines are therefore the bonds or valencies of the respective elements and we have:

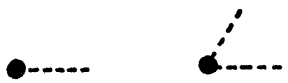

Hydroge

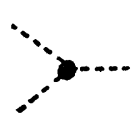

Nitrogen
Carbon

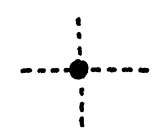

Thus a hydrogen atom is assumed to exist wherever a line ends. Oxygen is supposed to stand wherever a line makes an angle or two lines come together. Nitrogen exists at the point where three lines meet or arise, and carbon is thought to be at a point where two lines cross or four lines radiate. The length of the lines is immaterial. They are straight when representing single bonds and

1 Canadian Chemical Journal, Vol. 2, p. 135, May, 1918. curved when representing double bonds.

With these simple principles all organic structure-formulas can be accurately and exactly reproduced and the resulting geometrical figures or "organic symbols" offer certain advantages worthy of notice:

1. Compactness, for the structure has been brought to a very narrow compass, enabling the extensive use of the symbols where space is limited, e. g., in abstracts and catalogs.

2. Exactness, for each symbol represents only one definite organic compound of a definite structure and isomeric form.

3. Accuracy, for it is impossible to write for a given compound a structure symbol which is not theoretically correct, provided the simple rule regarding $\mathrm{H}, \mathrm{O}, \mathrm{N}$ and $\mathrm{C}$ is followed.

4. Clearness, for the design of the symbols of certain types of compounds is distinct and helps the student to remember the characteristic structure of a group of compounds or a radical like $-\mathrm{COOH}$, etc.

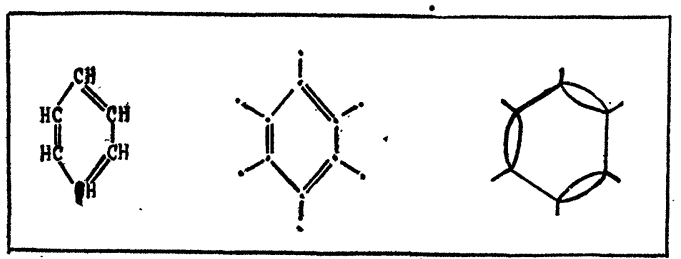

FIG. 1. The Organic Symbol for Benzene.

5. Simplicity, for with a very few rules thousands of compounds can be constructed and readily understood.

In Fig. 1 the comparatively simple transformation of the structural formula of benzene to its organic symbol is schematically represented, while Fig. 2 shows a comparison

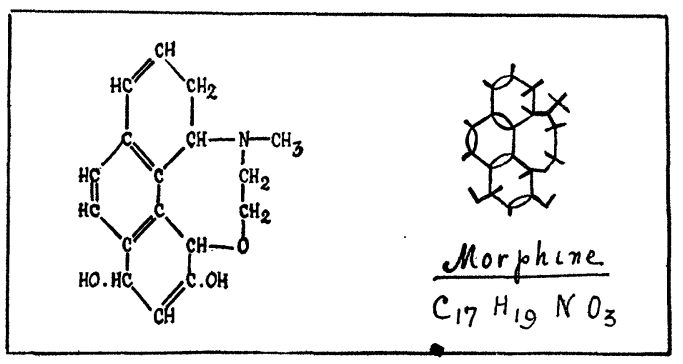

FIG. 2. The Organic Symbol for Morphine. 


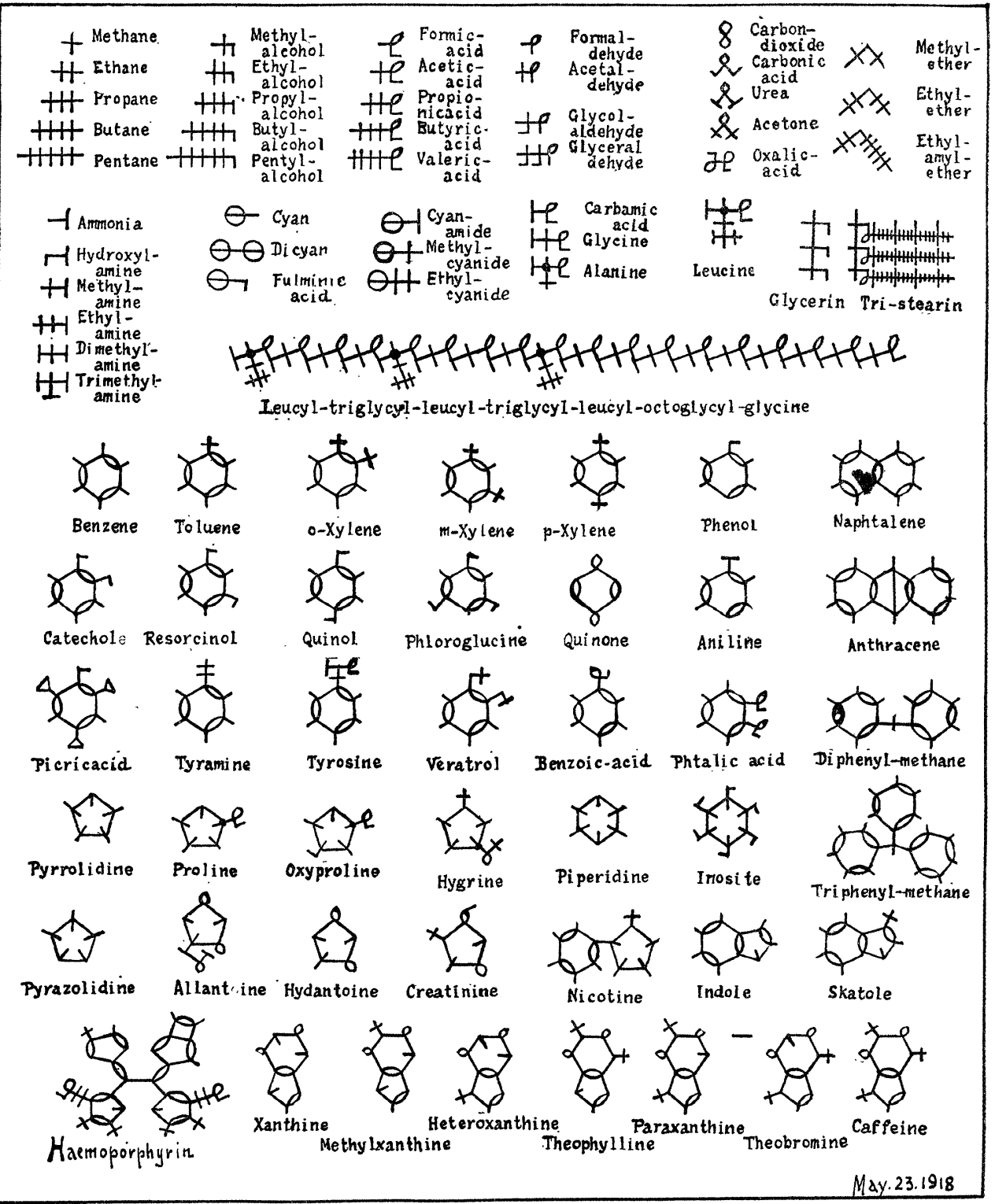

FIG. 3. Organic Symbols.

of the structural formula of morphine and its organic symbol. It will be noticed that as a whole the symbols differ not much from the structural formula as far as their geometrical form is concerned, but are naturally more precise. That is to say for example the symbol of acetic acid

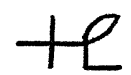

does not only mean $\mathrm{CH}_{3} . \mathrm{COOH}$, but actually

$$
\begin{aligned}
& \stackrel{H}{\dot{H}} \\
& \mathrm{H} \cdot \mathrm{C}: \mathrm{O} \\
& \dot{\mathrm{H}} \quad \dot{\mathrm{O}} \cdot \mathrm{H}
\end{aligned}
$$

and is therefore much more exact by recording not radicals but each atom separately. 
In Fig. 3 is a list of some typical symbols for different classes of compounds. A dot (e. g., in leucine) indicates an asymmetric carbon-atom and thus the optical active compounds are characterized. The long decorative design represents an octodeca-peptide or artificial peptone. Of the ring compounds mainly simple representatives have been selected, but some of the purin-bases at the bottom of the table (xanthin and derivates) show the simplicity of complex-rings. It will be noted that the different derivates are very plainly shown in their relationship, differing rangement of the atoms in the molecules. A ring of six atoms would be represented by a hexagon, and not by a square, one of five atoms as a pentagon and so on (compare, e. g., xanthin).

In certain cases nitrogen possesses a valency of five and in Fig. 4 the relationship of these symbols is shown. Also the use of the ordinary symbols in connection with organic symbols. $\mathrm{S}=$ sulfur in thiophen, $\mathrm{Cl}=$ chlorine in chloroform.

The writer has already employed the system in one of his classes with success and

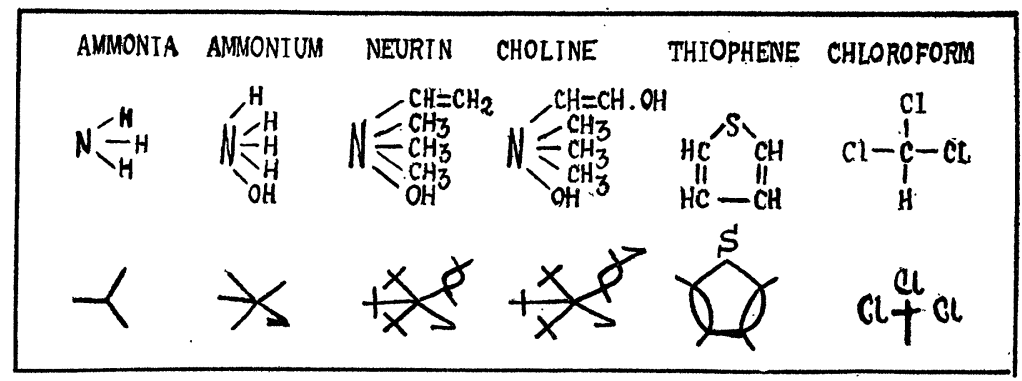

Fig. 4. Pentavalent Nitrogen and Examples of other Elements.

in that case only by the addition of one, two or three $-\mathrm{CH}_{3}$ groups (crosses in the symbols). Hæmoporphyrin, the mother substance of hæmatin of the hemoglobin of the blood, is an example of the more complex structures which recent investigations have disclosed.

With the rapid progress in organic chemistry and the structures of compounds becoming more and more complex, the need for a simple device of recording facts becomes apparent and $\mathrm{I}$ am indebted to the late Dr. Henry S. Denison, whose suggestions ${ }^{2}$ on a "chemical shorthand" caused the working out of the present system of "organic symbols." While the system is still in the precarious state of developmen!, it is necessary to warn against indiscriminate use of the principles involved in constructing symbols. An indiscriminate application would lead to confusion and for this reason certain standard types of symbols must be established. These standard types must or should conform as far as possible to the theories concerning the ar-

2 Denver Medical Times, Vol. 31, p. 360, 1912. found it a time- and space-saving medium in transmitting facts of organic chemistry to students and hopes that the system may become of value to other scientists.

INGO W. D. HACKH

Berkeley, Caljw.

\section{FRANK N. MEYER ${ }^{1}$}

Meyer was in the second year of his third great Middle Asiatic Exploration. His first trip of two years covered North China, including Mantchuria, in which province he walked 1,800 miles. His second trip of three years included the Caucasus, Persia, Turkestan, eastern Thibet, the middle districts of the great empire and Japan. His third trip was to have covered all the more southern portions of China likely to contain plants useful to western agriculture. During these seven years which were full of strange adventures he made thousands of interesting observations, penned

1 Drowned in the Yang-tsze-kiang, June 1, 1918, and buried in Shang-hai, China. 